(2) Open Access Full Text Article

\title{
A prospective comparison of phakic collamer lenses and wavefront-optimized laser-assisted in situ keratomileusis for correction of myopia
}

This article was published in the following Dove Press journal:

Clinical Ophthalmology

29 June 2016

Number of times this article has been viewed

\section{Gregory D Parkhurst ${ }^{1,2}$}

'Refractive Surgery Center, Carl R Darnall Army Medical Center, Fort Hood, ${ }^{2}$ Parkhurst NuVision,

San Antonio, TX, USA
Correspondence: Gregory D Parkhurst Parkhurst NuVision, 9725 Datapoint Drive, Suite 200, San Antonio,

TX 78229, USA

$\mathrm{Tel}+\mathrm{I} 2106159358$

Fax +I 2102490209

Email gregory.parkhurst@gmail.com
Purpose: The aim of this study was to evaluate and compare night vision and low-luminance contrast sensitivity (CS) in patients undergoing implantation of phakic collamer lenses or wavefront-optimized laser-assisted in situ keratomileusis (LASIK).

Patients and methods: This is a nonrandomized, prospective study, in which 48 military personnel were recruited. Rabin Super Vision Test was used to compare the visual acuity and CS of Visian implantable collamer lens (ICL) and LASIK groups under normal and low light conditions, using a filter for simulated vision through night vision goggles.

Results: Preoperative mean spherical equivalent was $-6.10 \mathrm{D}$ in the ICL group and $-6.04 \mathrm{D}$ in the LASIK group $(P=0.863)$. Three months postoperatively, super vision acuity (SVa), super vision acuity with (low-luminance) goggles ( $\mathrm{SVaG}$ ), super vision contrast (SVc), and super vision contrast with (low luminance) goggles ( $\mathrm{SVcG}$ ) significantly improved in the ICL and LASIK groups $(P<0.001)$. Mean improvement in $\mathrm{SVaG}$ at 3 months postoperatively was statistically significantly greater in the ICL group than in the LASIK group (mean change [logarithm of the minimum angle of resolution, $\operatorname{LogMAR}]$ : ICL $=-0.134$, LASIK $=-0.085 ; P=0.032$ ). Mean improvements in SVc and SVcG were also statistically significantly greater in the ICL group than in the LASIK group (SVc mean change [logarithm of the CS, LogCS]: $\mathrm{ICL}=0.356$, LASIK $=0.209 ; P=0.018$ and SVcG mean change $[\operatorname{LogCS}]$ : $\mathrm{ICL}=0.390$, LASIK $=0.259$; $P=0.024)$. Mean improvement in SVa at 3 months was comparable in both groups $(P=0.154)$. Conclusion: Simulated night vision improved with both ICL implantation and wavefrontoptimized LASIK, but improvements were significantly greater with ICLs. These differences may be important in a military setting and may also affect satisfaction with civilian vision correction.

Keywords: implantable collamer lens, LASIK, Rabin Super Vision Test, night vision, low-luminance contrast sensitivity

\section{Introduction}

Laser-assisted in situ keratomileusis (LASIK) has gained popularity as a safe and effective surgical method for correction of myopia. However, the method may not be suitable for high myopia or thin corneas, as a large amount of tissue ablation can produce more oblate corneas, thereby inducing higher order aberrations (HOAs). ${ }^{1,2}$

The Visian implantable collamer lens (ICL; STAAR Surgical Company, Monrovia, CA, USA) is a US Food and Drug Administration (FDA)-approved posterior chamber phakic intraocular lens (pIOL) for the treatment of myopia between -3.00 diopters (D) and -20.00 D. Since ICL implantation does not involve any tissue removal and leaves the central cornea untouched, ${ }^{1}$ it can benefit patients with high myopia or thin corneas 
who may not be suitable candidates for LASIK. Various studies have demonstrated the safety, efficacy, predictability, and stability of ICL for the correction of low, moderate, and high levels of myopia. ${ }^{2-4}$ Visual recovery time is similar to that of LASIK, 1-2 weeks. Further, a study comparing the outcomes of ICL implantation and wavefront-guided LASIK showed that ICL implantation induced significantly fewer HOAs and improved contrast sensitivity (CS). ${ }^{1}$ To date, however, no data have been published comparing the visual outcomes of ICL to wavefront-optimized LASIK.

LASIK outcomes have been studied specifically in naval aviators and found to be safe and effective. ${ }^{5}$ Recently, some US Army refractive surgery centers have been investigating ICL implantation in soldiers who desire less dependence on glasses and contact lenses and are not good candidates for corneal refractive excimer surgery. The purpose of this prospective study was to evaluate and compare night vision and low-luminance CS in subjects who had undergone either ICL implantation or wavefront-optimized LASIK.

\section{Patients and methods}

This is a prospective comparison study of 48 military personnel (34 males, 14 females) who underwent either ICL implantation (13 males, eleven females) or wavefrontoptimized LASIK (21 males, three females) for correction of myopia greater than $-3.00 \mathrm{D}$ (range: $-3.00 \mathrm{D}$ to $-11.50 \mathrm{D}$ ). Mean age was comparable between the two groups (mean age: $\mathrm{ICL}=27.6 \pm 7.3$ years [range $=19-46$ years], LASIK $=27.0 \pm 5.9$ years $[$ range $=20-41$ years ; $P$-value $=0.754)$. Further, there was no significant difference between the two groups in terms of preoperative spherical equivalent $(P=0.863)$. The mean preoperative spherical equivalent was $-6.10 \pm 1.76 \mathrm{D}$ (range: $-3.25 \mathrm{D}$ to $-11.50 \mathrm{D}$ ) in the ICL group and $-6.04 \pm 1.72 \mathrm{D}$ (range: $-3.00 \mathrm{D}$ to $-9.50 \mathrm{D})$ in the wavefront-optimized LASIK group. The mean scotopic pupil diameters in the ICL $(6.37 \pm 1.01 \mathrm{~mm}$, range $=4.5-8 \mathrm{~mm}$ ) and wavefront-optimized LASIK groups $(6.23 \pm 0.88 \mathrm{~mm}$, range $=4-8 \mathrm{~mm})$ were also comparable $(P=0.464)$. All procedures were performed at the Refractive Surgery Center of Carl R Darnall Army Medical Center. Each patient underwent a comprehensive preoperative examination for refractive surgery, including a slit lamp anterior segment examination, dilated eye examination, WaveLight Analyzer testing, corneal topography, and central corneal thickness (CCT) measurements via ultrasound pachymetry.

Patients who were good candidates for laser vision correction elected to undergo either LASIK or photorefractive keratectomy with the WaveLight Allegretto Eye-Q $400 \mathrm{~Hz}$ excimer laser system (Alcon, Inc., Hünenberg, Switzerland) with an optical zone of $6.5 \mathrm{~mm}$. The patients who elected to undergo photorefractive keratectomy were not included in this study. If patients were not considered good candidates for laser vision correction, they underwent additional testing to determine whether they were candidates for ICL surgery. The additional testing included confocal microscopy of the corneal endothelial cell layer, as well as intraocular lens master (Carl Zeiss Meditec AG, Jena, Germany), to determine the anterior chamber depth of the eye. If patients were found to be suitable candidates, they were offered ICL implantation. Patients who chose to undergo either wavefront-optimized LASIK or ICL implantation were recruited in the study, subject to meeting specific inclusion/exclusion criteria for the respective procedure.

\section{Inclusion and exclusion criteria LASIK group}

Inclusion criteria for the wavefront-optimized LASIK group included subjects with a stable refractive error as defined by a change of spherical equivalent of no more than $0.50 \mathrm{D}$ for at least 1 year, myopia of at least $-3.00 \mathrm{D}$ with no more than $3.00 \mathrm{D}$ of astigmatism, a corneal thickness $>500 \mu \mathrm{m}$, expected residual stromal bed thickness after LASIK of at least $300 \mu \mathrm{m}$ (assuming a $120 \mu \mathrm{m}$ flap thickness), and normal corneal topography. "Normal" corneal topography on these patients was defined as no abnormal posterior float elevation of the cornea, on Pentacam (Oculus Inc., Arlington, WA, USA); inferior/superior difference of keratometry readings of no more than $1.50 \mathrm{D}$ at $4 \mathrm{~mm}$ diameter from the center; and no thinning of the cornea associated with inferior steepening or posterior elevation in the same location of the cornea.

Subjects were excluded from the study if they had a history of dry eye syndrome, contact lens intolerance, or autoimmune disease. Pregnant or nursing females were also excluded. Finally, all subjects had to be available for follow-up at 3-month visit.

\section{ICL group}

To be included in the ICL group, subjects had to have stable refractive error for at least 1 year as defined by a change of spherical equivalent of no more than $0.50 \mathrm{D}$, myopia of at least $-3.00 \mathrm{D}$ with no more than $3.00 \mathrm{D}$ of astigmatism, an anterior chamber depth of at least $3.00 \mathrm{~mm}$, and healthy corneal endothelial cell layer as measured by confocal microscopy.

Subjects could not participate if they had glaucoma, cataract, iritis, pigment dispersion syndrome, pseudoexfoliation, or a prior history of any of these conditions. Pregnant or nursing females were also excluded. All subjects had to be available for follow-up at 3-month visit. 
Once patients were considered candidates for either LASIK or ICL surgery, written informed consent was obtained and they were enrolled in the study. The study had the approval of the Brooke Army Medical Center Department of Clinical Investigation review board and followed the tenets of the Declaration of Helsinki. To the extent that outcome may relate to the magnitude of preoperative refractive error, subjects were recruited to achieve a comparable distribution of myopic refractive error in the ICL and LASIK groups. An equivalent preoperative mean refractive error was achieved by ensuring that half of the participants in each group had a magnitude of myopia less than $-6.00 \mathrm{D}$, and the other half greater than $-6.00 \mathrm{D}$.

\section{Study parameters}

Once enrolled, patients underwent preoperative visual performance testing with the Rabin Super Vision Test (SVT; Precision Vision, LaSalle, IL, USA) to provide an operationally relevant measure of vision performance. ${ }^{6-9}$ The SVT includes high-contrast visual acuity and letter CS on a single chart. Subjects underwent SVT testing under normal and low-intensity light levels. Low-intensity light was simulated using a low-luminance night vision goggle (NVG) filter. SVT was conducted with best spectacle correction, first using the NVG with low-luminance filter, followed by SVT testing without the filter. This sequence (low-luminance NVG first) minimizes learning effects since visual acuity and CS improve with increasing light levels.

The parameters studied by SVT were super vision acuity (SVa), super vision acuity with goggles (SVaG), super vision contrast ( $\mathrm{SVc}$ ), and super vision contrast with (low luminance) goggles (SVcG). Patients underwent SVT with spectacle correction postoperatively at 3 months. Certified ophthalmic technicians, specifically blinded to which procedure the patient had undergone, carried out the testing.
Test results were also interpreted by an ophthalmologist blinded to the procedure.

\section{Statistical analysis}

Statistical analysis was performed using SPSS software (Version 17.0; SPSS Inc., Chicago, IL, USA). The data were examined for outliers using the outlier labeling method with a multiplier of 2.2. The Shapiro-Wilk test was used to check the normality of the data. Preoperative-to-postoperative changes were studied in the ICL and LASIK groups individually. To analyze the visual outcomes from baseline to 3 months postoperatively, paired $t$-test was used for normally distributed data; otherwise, the corresponding nonparametric test (ie, Wilcoxon signed-rank test) was used. Further, to compare the outcomes between the two groups, the independent $t$-test was used for normally distributed data; otherwise, the corresponding nonparametric test (ie, Mann-Whitney $U$-test) was used.

\section{Results}

A total of 48 patients ( 95 eyes) were recruited for the study. All the patients completed the 3-month follow-up period. There were no significant differences between the two groups in terms of age ( $P=0.754)$, race $(P=0.104)$, spherical equivalent ( $P=0.863)$, or scotopic pupil diameter $(P=0.464)$. Similarly, both the groups were comparable with respect to preoperative visual acuities: $\mathrm{SVa}(P=0.623)$ and $\mathrm{SVaG}(P=0.232)$ and contrast sensitivities: $\mathrm{SVc}(P=0.241)$ and $\mathrm{SVcG}(P=0.259)$. However, the mean CCT was statistically significantly higher in the LASIK group (CCT: ICL group $=547.30 \pm 46.36$, LASIK group $=571.29 \pm 38.07 ; P=0.007)$.

A statistically highly significant improvement $(P<0.001)$ in SVa and $\mathrm{SVaG}$ from preoperative-to-postoperative level was observed in both groups (Table 1). The mean change in SVa was comparable across both groups $(P=0.154$; Figure 1$)$. However, there was a statistically significant greater improvement in SVaG in the ICL group ( $P=0.032$; Figure 2).

Table I Summary of visual acuity and contrast sensitivity outcomes in the ICL and LASIK groups

\begin{tabular}{|c|c|c|c|c|}
\hline Variables & Groups ( $n=$ number of eyes) & $\begin{array}{l}\text { Preoperative } \\
(\text { mean } \pm \text { SD) }\end{array}$ & $\begin{array}{l}\text { Postoperative } \\
(\text { mean } \pm \text { SD) }\end{array}$ & $\begin{array}{l}P \text {-value (preoperative vs } \\
\text { postoperative comparison) }\end{array}$ \\
\hline \multirow[t]{2}{*}{ SVa (LogMAR) } & LASIK (n=47) & $-0.042 \pm 0.087$ & $-0.11 I \pm 0.069$ & $<0.001$ \\
\hline & ICL $(n=47)$ & $-0.033 \pm 0.089$ & $-0.126 \pm 0.070$ & $<0.001$ \\
\hline \multirow[t]{2}{*}{ SVaG (LogMAR) } & LASIK ( $n=48)$ & $0.083 \pm 0.095$ & $-0.002 \pm 0.079$ & $<0.001$ \\
\hline & $\mathrm{ICL}(\mathrm{n}=45)$ & $0.105 \pm 0.080$ & $-0.028 \pm 0.077$ & $<0.001$ \\
\hline \multirow[t]{2}{*}{ SVc (LogCS) } & LASIK (n=48) & $0.766 \pm 0.307$ & $0.975 \pm 0.255$ & $<0.001$ \\
\hline & $\mathrm{ICL}(\mathrm{n}=47)$ & $0.689 \pm 0.322$ & $1.046 \pm 0.212$ & $<0.001$ \\
\hline \multirow[t]{2}{*}{ SVcG (LogCS) } & LASIK $(n=48)$ & $0.266 \pm 0.229$ & $0.525 \pm 0.218$ & $<0.001$ \\
\hline & ICL $(n=44)$ & $0.20 I \pm 0.160$ & $0.59 \mid \pm 0.235$ & $<0.001$ \\
\hline
\end{tabular}

Abbreviations: ICL, implantable collamer lens; LASIK, laser-assisted in situ keratomileusis; LogMAR, logarithm of the minimum angle of resolution; LogCS, logarithm of the contrast sensitivity; SVa, super vision acuity; SVaG, super vision acuity with (low-luminance) goggles; SVc, super vision contrast; SVcG, super vision contrast with (lowluminance) goggles. 


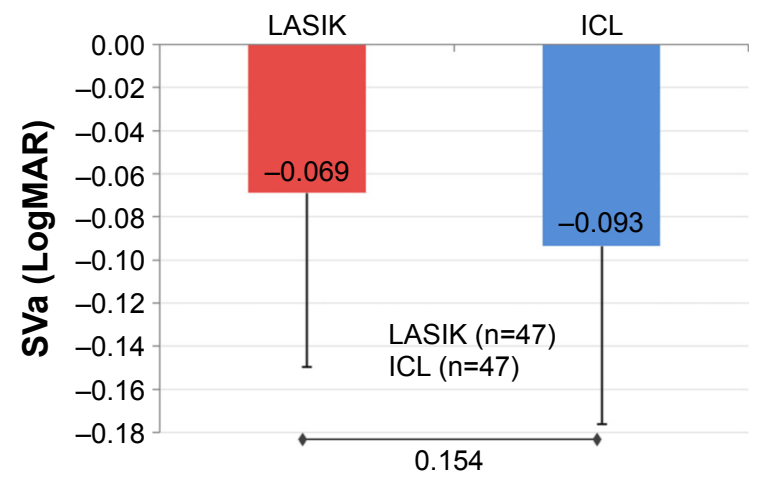

Figure I Mean improvement in SVa.

Notes: At 3 months, mean improvements in SVa (LogMAR) in the ICL and LASIK groups were comparable $(P=0.154$; error bars represent $S D)$.

Abbreviations: ICL, implantable collamer lens; LASIK, laser-assisted in situ keratomileusis; LogMAR, logarithm of the minimum angle of resolution; SVa, super vision acuity.

Preoperative CS (SVc and SVcG) was comparable between the two groups $(P>0.05)$. The postoperative improvement in SVc and $\mathrm{SVcG}$ from preoperative levels was statistically highly significant in both groups $(P<0.001$; Table 1). However, there was a statistically significant greater improvement in mean $\mathrm{SVc}$ and $\mathrm{SVcG}$ in the ICL group compared to the LASIK group ( $P$-values: $\mathrm{SVc}=0.018$ and $\mathrm{SVcG}=0.024$; Figures 3 and 4 ).

All surgeries were uneventful, and no vision-threatening complications were seen throughout the observation period.

\section{Discussion}

When deciding which refractive surgery procedures can be offered to a given patient, surgeons must evaluate a number of factors, including refraction, anatomy, other ocular

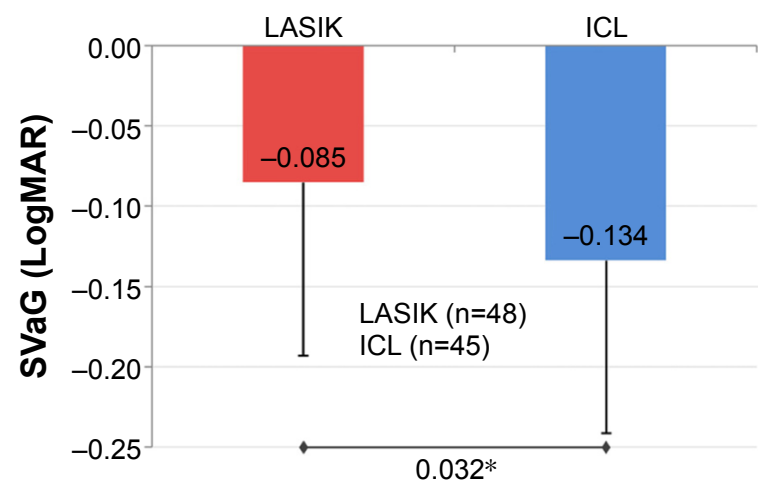

Figure 2 Mean improvement in SVaG.

Notes: At 3 months, mean improvement in SVaG (LogMAR) in the ICL group was statistically significantly greater than the mean improvement in the LASIK group ( $P=0.032$; error bars represent $S D$ ).

Abbreviations: ICL, implantable collamer lens; LASIK, laser-assisted in situ keratomileusis; LogMAR, logarithm of the minimum angle of resolution; SVaG, super vision acuity with (low-luminance) goggles.

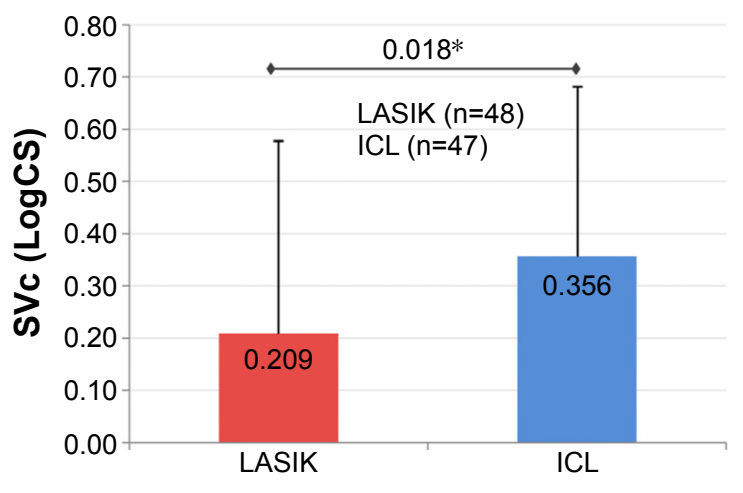

Figure 3 Mean improvement in SVc.

Notes: At 3 months, mean improvement in SVc (LogCS) was statistically significantly greater in the ICL group as compared to the LASIK group $\left(P=0.018^{*}\right.$; error bars represent SD).

Abbreviations: ICL, implantable collamer lens; LASIK, laser-assisted in situ keratomileusis; LogCS, logarithm of the contrast sensitivity; SVc, super vision contrast.

conditions and diseases, surgical recovery time, cost, safety and efficacy, and the patient's lifestyle and visual needs.

Excellent vision in a range of lighting conditions is highly desirable. This is particularly true in military combat, when excellent acuity and CS in low-luminance conditions are critical as soldiers navigate smoke, rain, dust storms, nighttime darkness, and other visually challenging environments. The inability to discern objects under these conditions could hinder operational performance and, at worst, can result in death. Although conventional LASIK has been shown to be a safe and effective means of correcting refractive error, diminished night vision and low-luminance $\mathrm{CS}$ are known potential complications of this procedure. ${ }^{10,11}$ There have been reports of postoperative glare and halos due to residual refractive error, decentered ablation, or the dilation of the pupil beyond the optical zone. ${ }^{12,13}$ The integration of wavefront technology

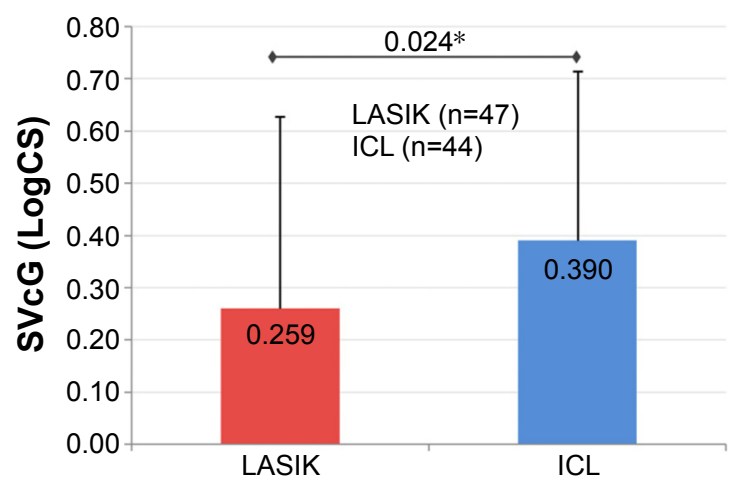

Figure 4 Mean improvement in SVcG.

Notes: At 3 months, mean improvement in SVcG (LogCS) was statistically significantly greater in the ICL group as compared to the LASIK group $\left(P=0.024^{*}\right.$; error bars represent SD).

Abbreviations: ICL, implantable collamer lens; LASIK, laser-assisted in situ keratomileusis; LogCS, logarithm of the contrast sensitivity; SVcG, super vision contrast with (low-luminance) goggles. 
into the LASIK procedure has improved visual outcomes, ${ }^{14,15}$ but a small proportion of LASIK patients still experience night vision disturbances after surgery. ${ }^{16,17}$

Night vision reports following ICL implantation have been promising. In the FDA clinical trial, the incidence of night vision problems such as halos, glare, and night driving difficulties decreased or remained unchanged for 3 years postoperatively. ${ }^{4}$ Moreover, ICL implantation did not result in any reported loss of CS at any spatial frequency, and a statistically significant improvement at six and 18 cycles per degree in mesopic CS without glare was observed. ${ }^{4}$ Reports by Sanders and Vukich have also shown ICL implantation to be safer and more effective than conventional LASIK in treating low, moderate, and high myopia. 3,18,19

A Cochrane review of studies comparing the outcomes of excimer laser refractive surgery with pIOL implantation for correction of myopia ranging from $-6.0 \mathrm{D}$ to $-20.0 \mathrm{D}$ also concluded that pIOLs are safer and more accurate than excimer laser surgical correction. ${ }^{20}$ Given the safety profile of pIOLs, the authors suggested that further trials comparing the efficacy of pIOLs with that of excimer laser refractive surgery for moderate myopia are needed.

In the current study, we analyzed and compared the visual acuity and CS outcomes in patients undergoing either ICL implantation or wavefront-optimized LASIK for the correction of moderate to high myopia ( $-3.0 \mathrm{D}$ to $-11.5 \mathrm{D})$. SVa improved significantly after ICL implantation as well as after wavefront-optimized LASIK (Table 1). NVGs present particularly challenging viewing conditions that include low luminance, loss of color discrimination, and diminished contrast. Simulating this field experience has been a useful and relevant measure of visual performance for military personnel. ${ }^{21}$ Although SVaG improved significantly in both groups (Table 1), ICL implantation was associated with statistically significant greater improvement in $\mathrm{SVaG}$ as compared to wavefront-optimized LASIK (Figure 2).

The improvement in both SVc and SVcG was also significantly better in the ICL group than in the LASIK group (Figures 3 and 4). Improvement in CS following ICL implantation has also been reported in the previous studies. ${ }^{1,4}$ Statistically significant worsening of CS at all spatial frequencies has been observed following wavefrontoptimized LASIK. ${ }^{16}$ For wavefront-guided LASIK, some studies have reported a decrease in CS after surgery, ${ }^{1,16}$ while others reported an increase..$^{17,22-25}$ Our current findings for ICL are consistent with previous studies. The improvement in CS from preoperative to postoperative level in the wavefront-optimized LASIK group may be due to any one of several factors, including varying levels of myopia, and the population in the current study. The induction of or reduction in HOAs may play a role in $\mathrm{CS}$ or night vision outcomes. However, the relationship between HOAs and visual outcomes is complex. Further research into the role of HOAs in night vision following the procedures described herein would be useful.

In addition to visual performance, surgeons may also have concerns related to lens opacity, ICL stability, or endothelial cell loss associated with the ICL implantation. Lens opacities have been a concern with ICLs due to their proximity to the crystalline lens. In the current study, no cataract development was observed; however, the 3-month follow-up period is too short to provide any conclusions about lens opacity. The literature suggests that while opacities were a significant complication with earlier iterations, ${ }^{26-28}$ they have been reduced to $<2 \%$ following design modifications. . $^{2,4,27-29}$

The stability of the implant is an important consideration, especially in the military setting, but they have been reported to be stable even after orbital trauma from a grenade explosion. $^{30}$

Finally, there are also concerns that ICL implantation could lead to endothelial cell loss. While endothelial cell count was not a variable in the current study, a review of the literature reveals that published results for endothelial cell loss following ICL implantation are varied. Different long-term studies have shown $6.57 \%$ endothelial cell loss at 2 years postoperatively, $6.09 \%-9.7 \%$ at 3 years, and $3.7 \%$ at 4 years postoperatively. ${ }^{2,4,31,32}$ Variations in patient demographics, sample size, and other factors may help to explain these differences, and further research may be warranted to better understand factors involved in cell loss.

\section{Conclusion}

In this study, low-luminance visual acuity and low-luminance CS significantly improved following ICL implantation, and the improvements were significantly better than those observed after wavefront-optimized LASIK. These findings support those of the FDA trial, which showed improved night vision performance following ICL implantation. Lowluminance visual performance is critical in military settings and may also affect satisfaction with vision correction in the civilian setting. Future studies with larger sample sizes may be useful to confirm these findings.

\section{Acknowledgment}

Jan Beiting (Wordsmith Consulting, Cary, NC, USA) and Raman Bedi, MD (IrisARC - Analytics, Research 
and Consulting, Chandigarh, India) provided research, statistics, and editorial assistance in the preparation of this manuscript.

\section{Disclosure}

The author is a consultant to STAAR Surgical, which provided professional assistance for the preparation of the manuscript and funded the publication processing fees. The views expressed in this article are solely those of the author. Department of the Army, Department of Defense, or the US government neither endorses nor is responsible for the opinions, interpretations, conclusions, and recommendations of this article. The author reports no other conflicts of interest in this work.

\section{References}

1. Igarashi A, Kamiya K, Shimizu K, Komatsu M. Visual performance after implantable collamer lens implantation and wavefront-guided laser in situ keratomileusis for high myopia. Am J Ophthalmol. 2009; 148(1):164-170.e1.

2. Kamiya K, Shimizu K, Igarashi A, Hikita F, Komatsu M. Four-year follow-up of posterior chamber phakic intraocular lens implantation for moderate to high myopia. Arch Ophthalmol. 2009;127(7):845-850.

3. Sanders D, Vukich JA. Comparison of implantable collamer lens (ICL) and laser-assisted in situ keratomileusis (LASIK) for low myopia. Cornea. 2006;25(10):1139-1146.

4. Sanders DR, Doney K, Poco M; ICL in Treatment of Myopia Study Group. United States Food and Drug Administration clinical trial of the implantable collamer lens (ICL) for moderate to high myopia: three-year follow-up. Ophthalmology. 2004;111(9):1683-1692.

5. Tanzer DJ, Brunstetter T, Zeber R, et al. Laser in situ keratomileusis in United States Naval aviators. J Cataract Refract Surg. 2013;39(7): 1047-1058.

6. Davies B, Rabin J, Gooch J, et al. Automated Testing of Super Vision and Night Vision Performance. Paper presented at: Annual Meeting of the Association for research in Vision and Ophthalmology (ARVO); May 2009; Fort Lauderdale, FL.

7. Rabin J. Contrast Sensitivity Review and the Super Vision-Night Vision Goggle Test. Paper presented at: Invited lecture at the Third Annual International Military Refractive Surgery Conference; January 2009; San Antonio, TX.

8. Rabin J, Davies B, Gooch J, et al. Super Vision: Quantification of Exceptional Acuity and Contrast Detection. Paper presented at: Annual Meeting of the Association for research in Vision and Ophthalmology (ARVO); May 2009; Fort Lauderdale, FL.

9. Rabin J, Gooch J, Davies B, Ivan D, Linnemeyer S, Foxworth M. The Super Vision-NVG Test. Paper presented at: Annual Scientific Meeting of the Aerospace Medical Association (AsMA); May 2009; Los Angeles, CA.

10. Pop M, Payette Y. Risk factors for night vision complaints after LASIK for myopia. Ophthalmology. 2004;111(1):3-10.

11. Villa C, Gutierrez R, Jimenez JR, Gonzalez-Meijome JM. Night vision disturbances after successful LASIK surgery. Br J Ophthalmol. 2007;91(8):1031-1037.

12. Macsai MS, Stubbe K, Beck AP, Ravage ZB. Effect of expanding the treatment zone of the Nidek EC-5000 laser on laser in situ keratomileusis outcomes. J Cataract Refract Surg. 2004;30(11):2336-2343.

13. Tsai YY, Lin JM. Ablation centration after active eye-tracker-assisted photorefractive keratectomy and laser in situ keratomileusis. J Cataract Refract Surg. 2000;26(1):28-34.
14. Kim A, Chuck RS. Wavefront-guided customized corneal ablation. Curr Opin Ophthalmol. 2008;19(4):314-320.

15. Lee HK, Choe CM, Ma KT, Kim EK. Measurement of contrast sensitivity and glare under mesopic and photopic conditions following wavefront-guided and conventional LASIK surgery. J Refract Surg. 2006;22(7):647-655.

16. Padmanabhan P, Mrochen M, Basuthkar S, Viswanathan D, Joseph R. Wavefront-guided versus wavefront-optimized laser in situ ker atomileusis: contralateral comparative study. J Cataract Refract Surg. 2008;34(3):389-397.

17. Tuan KM. Visual experience and patient satisfaction with wavefrontguided laser in situ keratomileusis. J Cataract Refract Surg. 2006;32(4): 577-583.

18. Sanders DR. Matched population comparison of the visian implantable collamer lens and standard LASIK for myopia of -3.00 to -7.88 diopters. J Refract Surg. 2007;23(6):537-553.

19. Sanders DR, Vukich JA. Comparison of implantable contact lens and laser assisted in situ keratomileusis for moderate to high myopia. Cornea. 2003;22(4):324-331.

20. Barsam A, Allan BD. Excimer laser refractive surgery versus phakic intraocular lenses for the correction of moderate to high myopia. Cochrane Database Syst Rev. 2014;6:CD007679.

21. Subramanian PS, O'Kane B, Stefanik R, et al. Visual performance with night vision goggles after photorefractive keratectomy for myopia. Ophthalmology. 2003;110(3):525-530.

22. Awwad ST, El-Kateb M, Bowman RW, Cavanagh HD, McCulley JP. Wavefront-guided laser in situ keratomileusis with the Alcon CustomCornea and the VISX CustomVue: three-month results. J Refract Surg. 2004;20(5):S606-S613.

23. Keir NJ, Simpson T, Jones LW, Fonn D. Wavefront-guided LASIK for myopia: effect on visual acuity, contrast sensitivity, and higher order aberrations. J Refract Surg. 2009;25(6):524-533.

24. Slade S. Contralateral comparison of Alcon CustomCornea and VISX CustomVue wavefront-guided laser in situ keratomileusis: one-month results. J Refract Surg. 2004;20(5):S601-S605.

25. Tuan KM, Liang J. Improved contrast sensitivity and visual acuity after wavefront-guided laser in situ keratomileusis: in-depth statistical analysis. J Cataract Refract Surg. 2006;32(2):215-220.

26. Sanchez-Galeana CA, Smith RJ, Sanders DR, et al. Lens opacities after posterior chamber phakic intraocular lens implantation. Ophthalmology. 2003;110(4):781-785.

27. Sanders DR, Vukich JA; ICL in Treatment of Myopia (ITM) Study Group. Incidence of lens opacities and clinically significant cataracts with the implantable contact lens: comparison of two lens designs. $J$ Refract Surg. 2002;18(6):673-682.

28. Sarikkola AU, Sen HN, Uusitalo RJ, Laatikainen L. Traumatic cataract and other adverse events with the implantable contact lens. J Cataract Refract Surg. 2005;31(3):511-524.

29. Kobashi H, Kamiya K, Igarashi A, Matsumura K, Komatsu M, Shimizu K. Long-term quality of life after posterior chamber phakic intraocular lens implantation and after wavefront-guided laser in situ keratomileusis for myopia. J Cataract Refract Surg. 2014;40(12):2019-2024.

30. McCauley MB, Anderson DM, Johnson AJ. Posterior chamber visian implantable collamer lens: stability and evaluation following traumatic grenade explosion. J Refract Surg. 2008;24(6):648-651.

31. Jimenez-Alfaro I, Benitez del Castillo JM, Garcia-Feijoo J, Gil de Bernabe JG, Serrano de La Iglesia JM. Safety of posterior chamber phakic intraocular lenses for the correction of high myopia: anterior segment changes after posterior chamber phakic intraocular lens implantation. Ophthalmology. 2001;108(1):90-99.

32. Pineda-Fernandez A, Jaramillo J, Vargas J, Jaramillo M, Jaramillo J, Galindez A. Phakic posterior chamber intraocular lens for high myopia. $J$ Cataract Refract Surg. 2004;30(11):2277-2283. 
Clinical Ophthalmology

\section{Publish your work in this journal}

Clinical Ophthalmology is an international, peer-reviewed journal covering all subspecialties within ophthalmology. Key topics include: Optometry; Visual science; Pharmacology and drug therapy in eye diseases; Basic Sciences; Primary and Secondary eye care; Patien Safety and Quality of Care Improvements. This journal is indexed on

PubMed Central and CAS, and is the official journal of The Society of Clinical Ophthalmology (SCO). The manuscript management system is completely online and includes a very quick and fair peer-review system, which is all easy to use. Visit http://www.dovepress.com/ testimonials.php to read real quotes from published authors. 